\title{
Live Action Role-Playing Games
}

Control, Communication, Storytelling, and MMORPG Similarities

\author{
Anders Tychsen \\ Michael Hitchens \\ Macquarie University \\ Thea Brolund \\ University of Technology Sydney \\ Manolya Kavakli \\ Macquarie University
}

\begin{abstract}
Live action role-playing games share a range of characteristics with massively multiplayer online games (MMOGs). Because these games have existed for more than 20 years, players of these games have a substantial amount of experience in handling issues pertinent to MMOGs. Survey and review of live action role-playing games, whose participant count can be in the thousands, reveal that features such as size, theme, game master-to-player ratio, and others interact to form complex systems that require several different groups of control tools to manage. The way that these games are managed offers a variety of venues for further research into how these management techniques can be applied to MMOGs.
\end{abstract}

Keywords: live action role-playing games; narrative; massively multiplayer online game; MMORPG; LARP; game master

$\mathrm{S}$ ome computer games model real-world activities, whereas other computer games attempt to model the processes of noncomputer games. For example, conflict-based board games and, of interest to this article, pen-and-paper (or tabletop) role-playing games (PnP RPGs) are directly reflected in their computerized counterparts such as Neverwinter Nights (2002 Atari/Bioware), Vampire the Masquerade: Redemption (2000 Activision), and Dungeon Siege (2001 Microsoft). Given the debates that have occurred as to the nature and role of narratives in computer games, it appears useful to study an example of an interactive narrative that does not involve computers.

PnP RPGs have been recognized as an important source of information for the study of interactive storytelling (Louchart \& Aylett, 2003; Peinado \& Gervás, 2004). PnP RPGs do not form a homogeneous group of games; however, they can be roughly described as an activity where one or more players take on roles within an 
interactive story, usually under the guidance of one or more game masters (GMs). This loose definition does not define the number of players or GMs (or the ratio of one to the other), the duration of the story, or the manner of the play itself. Nor does it define many other characteristics, allowing for significant variation between individual games.

Much of the attention given to PnP RPGs in the literature has focused on the style where a small number of players (typically four to six) sit around a table and the game is under the direction of a single GM. Many other forms of RPG styles exist. In this article, only those typically labeled-within the role-playing community-as live action role-playing games (LARPs) are examined. Whereas LARP itself is a term covering a wide variety of game play, it encompasses games that share a range of features with massively multiplayer online role-playing games (MMORPGs), notably,

1. physical action (avatar-based in MMORPGs)

2. high numbers of players possible in the game

3. low GM-to-player ratio

4. lack of a tight, narrative control

5. focus on player-player interactions

6. striving to provide an environment for emergent storytelling.

The lack of explicitly academic theoretical and empirical research within the field further hinders studies into LARPs, and most theoretical and practical knowledge is currently presented in nonacademic sources. However, recently, a few academics, especially in northern Europe, have begun to study LARPs as pervasive games and, in particular, applications of IT in these games (e.g., Söderberg, Waern, \& Åkesson, 2003). Large-scale freeform (see below) LARPs have existed for more than 20 years (Costikyan, 1998; Darlington, 1998-1999; Dollar, 2002; Söderberg et al., 2003). They have, therefore, had much longer to contend with problems pertinent to MMORPGs, for example, issues of trust, communication of the fictional world setting to the player, narrative control, and how to manage huge numbers of players with very limited GM resources. Even though the active medium is different-a physical as compared with a virtual world - it should be possible to utilize experiences from LARPs in the development of MMORPGs. In the words of Costikyan (1998), "A freeform LARP is as close to actual immersion in an interactive story that you'll get anywhere in the world." He recommended that computer game designers with an interest in interactive storytelling study LARPs. Likewise, Logas (2004) noted that "knowledge of live action role playing games . . . is very useful to players and designers of [MMOGs] because of the similarities in structure between them."

In this article, an overview of LARP history is presented, as well as a classification scheme based on surveys of LARPs, now and in the past 20 years. This includes how LARPs are planned and played and how the narrative development takes place. Core features such as GM control, fictional setting, and similar issues are discussed 
and their relative effect on various LARP types assessed. The review points to several venues for research that could benefit development and evolution of MMORPGs and indicate that LARPs form a prime source for inspiration in this regard.

\section{Defining LARPs}

The term LARP covers a wide variety of game variants, making them inherently difficult to define. Although the acronym LARPG has also been used, which explicitly includes the notion that game play is occurring, it is LARP that is the commonly accepted term in the gaming community. LARP is applied to gaming situations and not, for example, improvisational theater. Although activities such as the latter could be considered live action role playing, they are not games. In this article, the abbreviation LARP is used to indicate gaming activities, following consensus. In LARPs, players and game masters, combined, form the game participants, and the author of a LARP game or scenario does not necessarily equal the GMs - a person can write the manual for a LARP game without actually participating in it. The GMs are the participants who prepare, manage, and direct the LARP.

LARPs are RPGs, and defining LARPs can make use of the models arising from studies of PnP RPGs (see Edwards, 2001; Kim, 2003; Tychsen et al., 2005). LARPs follow the same terminology (role play, storytelling, rules, good play) and require the same in terms of player knowledge prior to game start: a character, the establishment of a fictional contract detailing the fictional world and how to act in and with it, and an opening situation (coined event zero for LARPs by Emrick, 2001). Similarly, an agreement on the conditions of the game (social contract) must still be achieved prior to game start to avoid conflicts later on. The following is not meant to be a complete definition of a RPG but does cover the most significant features.

1. The core of the game is role playing guided by rules. Each player takes control of one or more (although typically only one) character. A character is a fictional figure that the player tries to act (as role play).

2. The player will usually have full control of decision making at the character level. There is no author-audience relationship: Each player has a hand in developing a personal, perceived story.

3. The game is usually set in a fictional reality, which is communicated via the fictional contract. The contract is the shared understanding among the game participants of the game setting/world.

4. With very few exceptions, the games are supervised or guided by a GM, who assumes a variety of responsibilities depending on game type and style of play, notably, (a) facilitation of game flow, (b) environmental content, (c) administration of rules, and (d) engagement/entertainment. Some forms of LARP place very little emphasis on the GMs as guides and controllers of the games, and in these cases, GMs are delegated a role more akin to a player with some extra management functions. 
5. At least two participants are required. In general, noting the above exception, these will be a player and a GM. Typically, these roles are fixed, although in some games, the roles are interchangeable. The players and GMs together are the participants of the game.

The relative importance of these elements can vary. For example, a LARP can be very similar to improvisational theater, with only a few guidelines for rules and a very low-powered GM. In a computer RPG, the role of the GM is taken on (with varying degrees of success) by software.

LARPs can be viewed as forming a distinct category of RPG because of two unique features: (a) The players physically embody their characters, and (b) the game takes place in a physical frame. Embodiment means that the physical actions of the player are regarded as those of the character. Whereas in a RPG played by a group sitting around a table, players describe the actions of their characters (e.g., "I run to stand beside my friend"); in an equivalent situation in a LARP, a player would physically run to the appropriate point within the game space. The game space may range from a room to, in extreme cases, acres of countryside. These two features give rise to a wealth of design issues and complications when running a LARP.

These two features also produce an important consequence: Player-to-player interaction in a LARP often occurs without the presence of a GM. For a small group around a table, a GM will witness all such interactions. In a LARP, this is by no means the case, as, given the larger physical space, it is impossible for a GM to witness all such exchanges. The similarities between this and the situation existing in MMORPGs for player interaction should be obvious. On the other hand, most LARPs are normally designed on the assumption that all players will be present for most, if not all, of the duration of the game, an assumption that cannot be made for MMORPGs.

The nature of player-to-player interaction has important consequences for plot development and narrative in LARPs. As players are not constantly in the presence of a GM, the games cannot be designed so that narrative delivery and development for each player require constant GM attention. This also means that the games are typically designed so that only a subset of players is required to support a given plotline (unless the particular LARP has very few players), as their combined presence cannot be guaranteed. LARP players base individual narrative actions on a shared baseline of perception (i.e., the physical environment of the game, the fictional world setting, etc.). The players evolve perceived plots from a unified understanding of the game environment and the fictional world setting. Typically, LARPs are planned with several plotlines and these are typically of decentralized design so that they do not require constant GM control to develop-or they may not have any preplanned plotlines at all, being focused instead on producing an environment where plots emerge via the interactions of the participants and the characters they embody. There may be no central narrative (Lynch, 2000); however, more normal is that there is often a form of überplot: a plot involving or at least affecting the majority of the players. 


\section{Research in LARPs}

As most research into noncomputer PnP RPGs has focused on the traditional, smallgroup variety, research into LARPs is limited. Pertinent information occurs within a few books (notably, Bøckman \& Hutchison, 2005; Fine, 2002; Mackay, 2001; Montola \& Stenros, 2004; Murray, 1998; in general, Salen \& Zimmerman, 2003), a few journal articles, theses, Web articles, and manifestos (e.g., Fatland, 2005), however, first and foremost on the Internet. Web fanzines, discussion forums, resource sites such as the LARPer, Larplist, and Larp.com, as well as the Web sites of individual RPG societies and clubs form the mainstay of discussions. In recent years, however, an active interest in LARPS has arisen in the Scandinavian games research community. Efforts are currently focusing on studying LARPs in relation to mobile gaming, and the development of technological aids for pervasive gaming (Falk \& Davenport, 2004; Schneider \& Kortuem, 2001). Furthermore, LARPs form a possible source of information of relevance for interactive storytelling (e.g., Logas, 2004; Louchart \& Aylett, 2003).

\section{LARP History}

The origin of LARPs is uncertain and, to some extent, depends on how LARP is defined. The earliest documented live action variants of board games date back to 735 A.D., when games of chess were at times played with real people (Schneider \& Kortuem, 2001). Another example of live role play is the carrousel games from the European courts around the 17th and 18th centuries (Söderberg et al., 2003), which reconstructed battles, mythological tableaux, and the like. In the 1920s, psychoanalyst J. L. Moreno developed a form of live role playing, termed psychodrama or sociodrama, used for therapeutic treatment. This method employs guided dramatic action to examine problems of either an individual or group nature. The method was based in experiential methods, sociometry, role theory, and group dynamics and, to be safe for the participants, was strictly organized in nature (Söderberg et al., 2003). In more modern times, historical re-creations, such as Civil War battles in the United States or Viking villages in Scandinavia, have developed forms comparable with live action role play, however, they are devoid of game-like elements.

LARPs, as they are defined in this article, appear to have developed based on inspiration from tabletop RPGs such as Dungeons \& Dragons, more or less simultaneously in North America, Europe, and Australia sometime during the early 80s. Players from all of these continents claim to have hosted the oldest LARP, however, it appears that LARPs developed independently and with marked cultural differences. For example, in Europe, emphasis was placed on fantasy-themed, full-contact scenarios. North American scenarios were more focused on socialization aspects with science fiction themes, whereas Australian players focused on freeform-type games (see typology below). A LARP designed by Walt Freitag (cofounder of the Society for 
Interactive Literature) and entitled Rekon I was staged at Boskone (United States) in 1983 during a regional science fiction convention (Costikyan, 1998). Around the same time, Peckforton Castle in England hosted the LARP group Tresure Trap. This group was created by RPG players who had taken their game play to a physical dimension, eventually gaining permission to use Peckforton Castle (Dollar, 2002). In Australia, the first freeform LARP was organized at a games convention entitled Octocon, organized by the Canberra Wargames Society in October 1982. The scenario was written by Peter Quinton and based on the Traveller (2002 Quiklink) PnP RPG system. Character interaction was structured around rank and specialization, and players developed their own characters and character goals. In the following years, the Australian freeform LARPs grew in player number and developed more structured characters, preplanned personalities, and intercharacter goals (J. P. Hughes, C. Walker, et al., authors of the aus.games.roleplay group, personal communication, August 2005). In parallel with the game-based LARPs, educational LARPs have been developed for teaching purposes, for example, and LARPs have proven a useful testing ground for pervasive gaming technology (Schneider \& Kortuem, 2001).

A diversification similar to the one in PnP RPGs occurred in the LARP environment during the late 80s and into the 90s. With the rise of horror and cyberpunk PnP RPGs in the 80s and early 90s, LARP scenarios set in these genres emerged. With the rising popularity of Vampire the Masquerade (VtM; 1991 White Wolf Games), a PnP RPG that has a gothic present-day setting where players take the roles of vampires, a new form of LARP with gothic themes became popular. These new LARP forms provided a less combat-oriented type of LARP than the large-scale fantasy LARPs. A major problem for the cyberpunk, horror, and sci-fi forms is the issue of generating a believable environment. Although Europe sports numerous castles and ancient woods perfect for fantasy games, the continent is less heavy on nuclear wastelands or mega-cities. Factories, old forts, and even submarines have been used to generate the setting of such events, however, they remain in the minority and usually cater to a maximum of 50 players because of the problem with developing an environment consistent with the world setting. Mafia-themed hardball (BB gun) games were also developed, as were similar realism-focused games based on computer simulations (Dollar, 2002). An alternative is to require the players to exercise considerable imagination in considering a basically empty physical environment (such as a hall) to be the posited setting of the game. This allows for a diverse range of game settings, including science fiction, the heavens and hells of various religions, and even the human body, where players take on the roles of various diseases and bodily defenses. This can also allow for fantasy settings in the absence of a convenient castle. In comparison, LARPs of a type similar to LARP VtM are somewhat easier to stage, as the game takes place in the real world. This was one of the most important factors in making LARP VtM fairly widespread and popular, along with the popularity of the PnP form of the game. The focus on noncombat and intrigue also attracted players from the RPG environment who otherwise did not play fantasy LARPs. 
Today, LARPing is a widespread hobby, especially within the United States and Europe, and caters to at least 100,000 players worldwide (Schneider \& Kortuem, 2001). Games range in size from a handful to more than 4,000 players at the annual event of the Lorien Trust LARP organization in England. Exclusively, the large LARPs with hundreds or more players are set in fantasy/medieval world settings, which is the historic genre for LARPs.

Professional, full-time LARP sites also exist in England (e.g., the Labyrinth outside London). Around the hobby, a small industry has grown-up supplying latex weapons, costumes, theater props, and special effects, and numerous special effects and make-up artists work in the LARP environment for training purposes. The biggest LARPs are week-long scenarios that run pervasively during this time, with up to 4,000 participants-fully costumed by professional costume makers-with the castles and forests of rural England forming the backdrop of fictional worlds. In these games, teams of GMs and nonplayer characters (NPCs) use modern communication technologies to keep track of events. In short, LARPs are as close to MMORPGs as is possible outside the virtual medium of these games.

\section{LARP Classification}

To date, there are no published models of classifications of LARPs, barring various category-based observations published on the Internet (Emrick, 2001), or divisions that only account for certain aspects of LARP (e.g., Logas, 2004), or publications on LARPs from a variety of angles from the Knutepunkt conferences held in Scandinavia, Finland, and Denmark (e.g., Bøckman \& Hutchison, 2005). It has not been possible to locate any models published in the academia either; however, this is not to say that they do not exist. Lacking empirical data on which to base a classification model, what is attempted here is to map all possible variations of LARPs. This is done by mapping the variance of a range of core features (duration, size, etc.). The classification model that arises from using the categories of each feature should cover all known forms of LARP. From a top-down perspective, the treatment of the features forms an axis for the classification of LARPs.

\section{Duration}

Most LARPs are either scenarios (or single-shots) or campaigns (also known as chronicles; Logas, 2004). Both types are pervasive within their defined time frame. Scenario LARPs are stand-alone events, complete in themselves, and are the typical type encountered at game conventions. Their duration can vary from a few hours to a weekend or even a week. Irrespective of temporal duration, there is usually a defined beginning and end of the story line. The end is usually climactic. A scenario can be run with breaks, in which the individual in-game periods are called sessions - as in a PnP RPG "game session” (Emrick, 2001). 
Campaign-duration LARPs are made up of two or more separate LARP events (also termed sessions or scenarios). The individual scenarios of a campaign may end with some form of mini-climax to define the end of play, in a manner loosely equivalent to the end of stand-alone scenarios. The separation between scenarios of a campaign need not be temporal, but often is. An example is the long-running English fantasy campaigns and their equivalents in Denmark and Sweden, where people meet, for example, once a month or four times a year in the physical frame of the game and continue the game where they left it. Between the scenarios, there can be meetings where people discuss the unfolding plot, plan new interactions or other activities, or even role play necessary encounters. Intermittent meetings can therefore range from practical planning sessions to mini-LARPs subservient to the main campaign. Campaigns may be open-ended, with no predefined conditions that bring the game to an end, or they may include a limited number of linked scenarios, normally with a predefined climactic ending. These can feature minor climaxes during the individual scenarios and can be termed "cyclically climactic." Fixed-length campaigns typically feature stronger GM control than open-ended campaigns.

LARPs of any duration are usually followed by a game wrap (Emrick, 2001), where the game is rehashed and players can comment on the game, plots, intrigues, and each other. In campaigns, this period is also used to update ongoing plots after each scenario.

\section{Size}

The minimum number of participants in a LARP is two (generally one player and one GM), whereas the theoretical maximum is undefined. The largest actual number of participants in a single LARP event to date is around 4,000 (Dollar, 2002; Falk \& Davenport, 2004), a game that was organized in Southern England by Lorien Trust. The number of participants in a LARP is important for how the actual playing is conducted, for example, in how players are kept entertained, storylines are developed, and scenarios are planned. These issues will be discussed below, where the game sizes with which they are commonly associated will be identified.

Related to number of participants is the GM:player ratio. This is relevant to the amount of control that can be asserted by the GMs. Although the GM:player ratio can vary (e.g., a 20-player LARP may have 2 or 6 GMs depending on the particular game), as a general rule, the relationship between number of players and number of GMs is inversely exponentially proportional. This is mainly due to the practical considerations of recruiting sufficient GMs and maintaining a sufficient level of communication between them during game play. An important LARP size is the here termed ultra-LARP (ULARP). The term ultra is used to follow MMORPG terminology, where a 10,000+ player server (or shard) is termed an ultra-MMORPG (UMMORPG). The exact point at which a LARP becomes a ULARP is debatable, but it requires at least approximately 100 players and a low level of GM control. 
Because the workload involved in generating a controlled experience for more than 100 players is beyond the time available to most LARP authors, ULARPs tend to have a limited preplanned plot with very low GM:player ratios and well-developed world settings (facilitating the creation of emergent content and an open-ended game style). These games are inherently nonlinear with respect to the narrative development, as each player, more or less, is responsible for his or her own entertainment and for forging his or her own destiny and story in the games. Usually, GMs use an information network to maintain an overview of the game and affect or direct events in various situations. The employment of specially empowered players in core roles, typically leader positions, provides an elongated hand to the GMs.

The ULARP situation is similar to that of a MMORPG, where players can outnumber GMs by a factor of 100 or more. ULARPs are usually open-ended or cyclic campaign-type games and appear to have the largest following of LARP players in the world. In very large LARPs, there will usually be a location where GMs and other game controllers meet and keep props and other equipment. This location is termed a GM central by Emrick (2001).

\section{Rules}

The rules of LARPs can be subdivided into several categories, depending on whether they cover physical interaction, verbal interaction, and so on. Often, rules for combat are viewed as a category separate from all other rules, because combat is the primary rules-mediated means of interaction between the players. The rules for interaction in a LARP are usually much simpler than in the average tabletop RPG (Costikyan, 1998) — to avoid confusion and time-consuming GM arbiting, they need to be easily understood. Terms such as light and heavy are generally used to differentiate rules systems. The rules of a LARP are, in practice, less flexible than in PnP RPGs because of the lack of a GM to sanction bending or breaking of the rules. This is especially true for large LARPs, which require simple, inflexible rules systems.

Combat in LARPs is defined as any simulated fight or other physical conflict between the characters and is one of the primary means of generating tension. Simulated combat is usually guided by a rule system covering, for example, weapon damage. Combat ranges from rock-paper-scissors-like systems to full steel. Following martial arts terminology, four subdivisions have been in popular use in LARPs: noncombat, noncontact, semicontact, and full-contact. In the highly active Scandinavian LARP scene, a large variety of rules governing physical interaction has been developed, which extend beyond that of combat.

Combat in LARPs can be handled at the discretion of the players or be monitored by a GM. Some LARPS have GMs whose primary responsibility is arbitrating combat (combat GM).

The potential nonpresence of a GM in a given in-game situation means that trust between players is important. Because different people play LARPs for different reasons, 
incidences where the trust is broken-player versus player ( $\mathrm{PvP})$-oriented play and similar conflicts-occur. However, the fact that the player cannot, for example, hide behind a virtual account as in a MMORPG does mean that punishment is easier to mete out. This is possibly why trust issues play a significantly smaller role in LARPs than in MMORPGs. In general, the fewer the number of participants, the fewer the problems; on the other hand, the more participants, the greater the diversity.

\section{World Setting and the Character}

The world setting or framework for the fictional world in which the game takes place varies greatly in LARPs. Generally, these can be divided into either real-world settings or fictional settings. The latter category can be further subdivided into two categories. (a) Themed world settings, where the game space is modified to accommodate a theme, for example, fantasy-world games where theater-style props are used to improve the immersive qualities of the game environment. Examples include using costumes, medievalstyle weapons, and factories as backdrops for cyberpunk-themed games. (b) Visualized world settings, where an unmodified real-world setting is used as a shared space for the imagined setting, with little or no support in the form of props and costumes.

The large open and freeform-type scenarios that have been running in Scandinavia and England for the past 15 to 20 years usually have very well-developed world settings, with NPCs forming part of the environment. The characters are generally planned by the players, and it is up to them to integrate them. In contrast, smaller LARPs usually have less well-developed world settings, because of the decreased need for a large-scale fictional world, focusing predeveloped material at the level of the individual character or group of characters. This means that the character-level material is usually much more developed, however, only areas of the world setting pertinent to the scenario in question are covered.

The level of background information provided to the players forms an important role in the feel of a LARP. A well-developed world setting provides information to the players on how to act in the game and helps the establishment of the fictional contract. Furthermore, the background provides plot in itself: If a player knows that his or her kingdom is at war with the neighboring one, he or she will be wary of a character from that area, thus providing a basis for conflict in the game and thereby the development of plot. These kinds of game-intrinsic plot developments generally operate at the group level, in this case, the two groups of players whose characters belong to the two different kingdoms. However, the background also has the potential to generate deeply personal plots, for example, in the forging of a friendship between two player characters from the warring kingdoms (a friendship that can be transferred to the real world, outside the game). Individual character development may be limited to a name and brief description or be an extensive document on the character's personality and background. Although greater detail allows for more personalized plot development, this is not always used. It is then simply there for "color" as in a number of existing MMORPGs. 


\section{Control and Management of LARPs}

Because players can have a large degree of control over their characters and the game in general, LARP authors and GMs face substantial challenges in controlling —or, more specific, managing - a LARP. As mentioned by Logas (2004), LARPs are, to a large degree, about not controlling the players but rather managing the entire LARP situation, however, this does not mean that the GMs are unable to affect the focus of the players.

Apart from the organizational challenges (creating props, acquiring a physical game space, etc.), when planning a LARP, the author/GMs are faced with two primary planning and management challenges (unless the scenario is completely open): (a) What are the players supposed to be doing? and (b) How are they supposed to do the things they should do? (accomplish in-game goals, etc.). These two challenges are, respectively, addressed via five overall groups of control functions, which form the range of tools available to authors and GMs in managing a LARP scenario (see Figure 1):

1. basic control functions

2. number and type of in-game events

3. character generation

4. preplanned character objectives, which encourage the players to focus in certain directions

5. the division of temporal control

Control functions in LARPs can also be described according to whether they operate before the game begins, during, or both and whether they operate in-game or out-ofgame. For example, the use of mobile phones to communicate is an out-of-game GM control tool. In comparison, the staging of events to promote certain plots is an in-game tool.

\section{Basic Control Functions}

Control in LARPs can be viewed as a bottom-up process, with the basic control functions being at the bottom. These are management tools associated with all types of LARP, even the completely open, which merely supply a game world and a rule set for the players to use for their own entertainment. Four general categories belong to the basic control functions: (a) GMs, (b) world setting, (c) NPCs, and (d) rules (either light or heavy) (see Figure 1).

In practice, a LARP requires all of these features, barring NPCs, which are, however, a regular feature of LARPs. GMs are (usually) necessary to manage and run the game. NPCs act as the proverbial eyes and ears of the GMs (i.e., they form an ingame information gathering-and-control network) and are primary producers of ingame content. Rules and world setting are discussed above. 


\section{Figure 1}

\section{Control or Management Functions in Live Action Role-Playing Games (LARPs)}

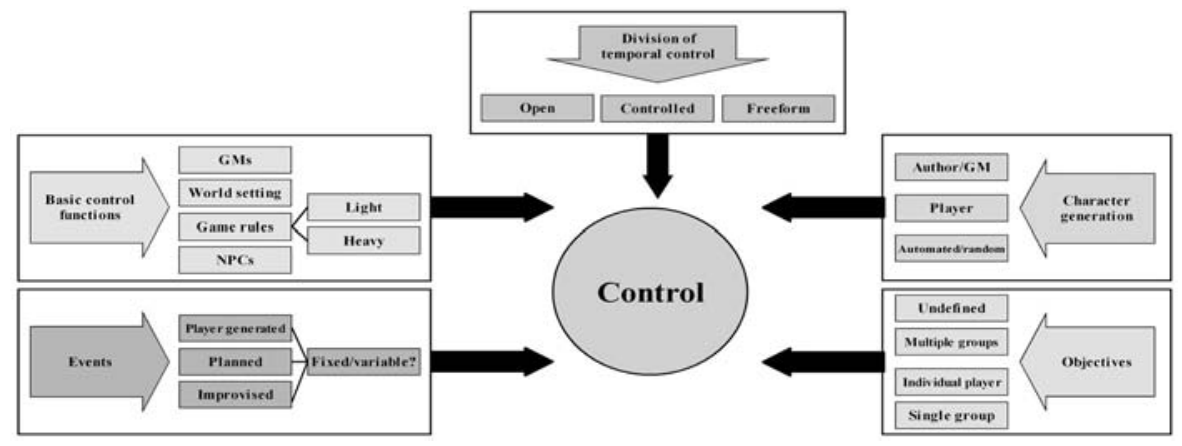

Note: GMs = game masters; NPLs = nonplayer characters. Five overall groups contribute to the control in LARPs: objectives, events, character generation, division of temporal control, and basic control functions.

\section{Character Objectives}

In a typical PnP RPG, the characters are working toward some immediate goal or objective, based on a current game session or quest, but usually also have long-term goals or incentives (Fatland, 2005), which can change as the game progresses. These may be to accumulate money, rescue the princess, defeat the supernatural horror, or any other goal present in the full range of literature. As may be expected of what is in most cases an amateur hobby, the terminology in common use is less than precise and the target of the aspirations of the character may be termed goals or plot. Here, the term objective is employed to describe all of the above.

LARPs can demonstrate the same variety as PnP RPGs, both in the setting/genre (e.g., horror, fantasy, science fiction, real world) and in the focus of the action (e.g., combat, political intrigue, or investigation). In all PnP RPGs, the GM (or other author of the session) has a choice as to how much preparation will be put into laying out a game session, both in planning a plot sequence and in setting objectives for the characters. This may or may not be done in concert with the players and is in addition to the development of a background and a world setting. The amount of prepared material can range from the encyclopedic to the nonexistent. The players may decide on objectives for their characters independent of the GM and these are then worked into the game as it progresses.

In a traditional PnP RPG, the GM is constrained by having both a small number of players (typically up to around six) and by the players expecting to work as a team 
toward a common objective. In a LARP, the much greater variation in participant number (size) allows, or in some cases forces, the GM to consider different options. Four categories of objective preparation for LARPs are here identified. In theory, these categories are orthogonal to participant numbers, but in practice, they are applied to games of recognizably different size categories. The categories are distinguished by whether the playing group is treated as a homogeneous unit or not and whether objectives are individual to characters or are provided at the level of groups of players (see Figure 1).

1. Undefined: The world setting for the game is defined in such a way as there are no obvious conflicting groups. Characters are not given any individual objectives. Players may form their own groups, but these are also left to their own devices. The players must find their own entertainment within the game. This type of LARP requires a detailed world setting. However, even a 100-page definition of the world is less effort to write than 1 page for each character if participant numbers are in the hundreds or even thousands. As such, this style of game is most suitable for very large numbers of participants. This category is well suited to open-ended play.

2. Single group: All participant players are considered a single unit and are intended to work cooperatively toward a common goal. Characters may have individual objectives and these may conflict with those of other characters, but such conflict is not intended to prevent the group from achieving its main goal. This category describes the typical situation in PnP RPGs. The size of the playing group to which this can be applied in practice is relatively small, typically in the single figures. Keeping a larger group focused on a single goal is difficult, especially for a single GM. Although the use of multiple GMs in practice can increase the player numbers into the low teens, this leads to group fragmentation and difficulties in having each subgroup contribute meaningfully to the shared objective.

3. Multiple groups: Objectives are no longer set at the level of the entire player body. Instead, characters are grouped together (typically in line with the world background of the game) and objectives are defined for these groups. Typically, the groups will be competing factions, conflicting politically and/or militarily. Character membership in a political faction will define their main objective. Within this, the players are expected to generate their own interactions and entertainment and to set goals for their individual characters. Examples of this include LARPs where players choose membership in competing factions of vampires. The amount of preparation required for a game of this style is more than that required for a singlegroup approach but less than the following category. Generally, multiple-group games can have very large participant numbers (in practice, thousands).

The above three types of objective preparation are closely related to existing MMORPGs, both in terms of (potential) player numbers and in terms of the lack of game-provided individual character objectives. For example, in World of Warcraft (2004 Vivendi Games), there are eight defined races from which the players can choose to create characters. These are grouped into two opposing factions (the 
Alliance and the Horde). However, the quest system does not define any personal, unique objectives to players, although there is a limited number of character classspecific quests.

4. Individual player: This category can be viewed as an extreme form of the multiplegroup approach, with the groups numbering one player in size. Rather than having the entire body of players working toward a shared goal, players can be provided with different, and possibly conflicting, goals. Each character has its own set of objectives. Some of these may correspond with the objectives of other characters, leading to the formation of cooperating subgroups within the entire playing group. Group objectives are, however, typically generic (defend the city, achieve political power) and limited in number, whereas individual objectives can be quite detailed. These objectives can range from political groupings, which last for the duration of the game, to temporary groups in the pursuit of a single, limited objective. The jump from single group objective to the individual player level significantly complicates the communication patterns within the game (to an extent, that is worthy of independent study). There is a distinct change in the feel of a LARP between this category and the former, as it is difficult for a GM to interact simultaneously with players seeking to achieve different objectives. In practice, it is normally difficult to provide sufficient GMs to allow one GM for every goal that a player or group of players is currently seeking (unlike the above, where the single group objective can be served by a single GM). The objectives in this style of game are commonly prepared in such a manner that many can be accomplished via player-to-player interaction, with limited or no GM involvement. The amount of preparation required to provide sufficient plots for each player to fill the game session in an entertaining manner places a practical (although not theoretical) size limit on the games. Typically, this is around 50 participants, rarely more than 200 .

\section{Event Control and Division of Temporal Control: Managing Players and Narrative Development}

The type and application of in-game events, how they are controlled and planned (e.g., Fatland, 2005), and the amount of in-game time these cover are closely related. Therefore, event control and the division of temporal control are treated jointly here.

The concept of an event is here broadly defined to include any in-game situation that the author and/or GMs create or affect. Although this definition may have to be modified in future treatments, it is useful in this review. Events can be either (a) preplanned, (b) improvised, or (c) player generated. For example, the author of a scenario may have planned for a battle to take place between the orcs and the elves in a fantasy-themed scenario. During the LARP, the GMs decide that the battle should be followed by an ambush conducted by trolls on a surviving group of elf players. Improvising events is a form of micromanagement of the LARP situation, and even preplanned events are often modified as the game unfolds, because LARPs can move in directions unforeseen by the author due to the freedom of the players (expect perhaps the controlled-type LARPs 
discussed below). In-game modifications are possible due to the presence of live GMs and their ability to affect the physical game space and players directly-unlike MMORPGs, where the ability of GMs is more limited due to the nature of the virtual medium and the tool sets they are provided. Player-generated events are not directly related to GM control issues, because they are generated outside the direct influence of the GMs; however, they can form handy tools for GM manipulation.

Events are directly related to the narrative development of the game. In the case of the battle described above, the outcome of this is essential for the further development of the game narrative (which is here loosely defined as the sum of stories experienced by each individual participant).

The level of control that GMs have on events is largely dependent on whether the outcome of the events is fixed or variable, controlled by player actions. For example, if the GMs are making sure that the elves win the battle, the event has a fixed outcome and cannot be affected by the players. Variable outcome events make control and management more difficult, however, player freedom is preserved. Variable outcome events can be restricted to a number of possible outcomes, either defined by the author/GMs or the nature of the event (e.g., either the orcs or the elves win, or there is a draw).

Concerning temporal control in LARPs, the amount of scenario time that is spent by the players in taking part of preplanned or improvised events is a direct way of measuring the level of control in a LARP scenario. GM control can range from the extremely tight, with each scene the players experience being a highly preplanned event, to the GMs doing little more than applying the rules to resolve attempted player actions. Three broad categories are here identified based on the temporal distribution of control. These categories are orthogonal to character objective categories, giving 12 potential combinations. However, in practice, controlled-type LARPs are usually affiliated with single-group (generally small size) or individual objectives games, whereas freeform and open LARPs are usually affiliated with undefined, single-group (generally large size) or multiple-group objective approaches.

1. Controlled (also termed multiform): The GMs control developments very tightly. Usually, this means a planned development of events in the game, following either a linear path/narrative or one that is multilinear with plot points and a set climax or similar. Under the main plot, several nonlinear narratives may be developed at the character level. This type is the closest LARP analogy to tabletop RPGs, and most LARPs in this category have player numbers in the single figures. It is a type of game also often used in a professional or educational context (e.g., leadership, team building, and survival courses).

2. Freeform: There is no structured, preplanned narrative to which the GMs are working. However, the GMs exert some narrative control via a limited number of in-game events (e.g., via implementation of an overarching plot or überplot). This may involve (or at least be witnessed by) all participants or a subgroup of the players, depending on the nature of the event. The GMs may also provide limited narrative-related information to 
the players, for example, by planting rumors. Apart from these limited elements, the GMs provide no direct narrative guidance. The GMs may assign monsters and other opponents and challenges to harass the players. They also resolve player actions and disputes, as requested and required, and handle practical issues such as communication, food, and so on. A possible variant of this form of LARP, coined dissociative LARP (T. Harviainen, personal communication, April 2006), has been proposed. In this form, the GMs also have no control over narrative flow, character objectives, and so on, but they have the in-game power to define the outcome of any event (they, of course, have to be aware of the event happening).

3. Open (also termed freestyle): The open form is superficially similar to the freeform. There are no preplanned events in this category and information provided has no direct narrative relation. GM-provided challenges are likewise simply for entertainment purposes, not for advancement of a preplanned narrative. It can be seen that this style is well suited to the undefined objective preparation category in the previous section and can be applied to the others from there as well. This form of LARP does not have a fixed outcome.

The freeform, freestyle, and open LARPs are traditional and broadly used terms in the LARP environment and have generated a large number of different definitions. In this article, the terms are precisely defined, and these definitions will vary with some of those in the LARP community. Here, the terms freeform, open, controlled, and so on are defined on the basis of the division of temporal control between the players and the GMs. The GMs can, in this instance, be either actual GMs or any other nonplayer, who control in-game events for a certain amount of time.

\section{Character Generation}

The character generation process directly affects the level of control that GMs have in a LARP, for example, through the generation of motivations for player behavior. If the players control this process, the GMs are harder pressed to know how players will act in given situations, whereas predefined characters and possibly character goals ensure a better GM knowledge of the characters and thus better control.

Character generation, although not strictly dependent on event control, is in practice often related to this feature of LARPs. Open-type LARPs typically have players generate their own characters. Freeform LARPs can use either this option or authorgenerated characters, whereas controlled scenarios typically have author-defined characters, because of the increased need to control player focus and interactions (e.g., via predefining player objectives).

\section{The Physical Game Space}

In LARPs, the use of the physical game space in which players physically carry out the actions of their characters is not without its problems. Authors are faced with the 
choice of whether to display the fictional world setting in the real world or leave the details to the imaginations of the players. The former choice obviously lends believability but has various practical problems in its implementation, whereas for the latter, the issues are reversed. Similar considerations apply to how players are to handle fantastical elements of the world setting, such as altered physical parameters like spell casting. Financial funding is probably the factual limiting factor in this regard, as this limits the variety of props, technologies, costumes, and so forth. For example, it is difficult to create a proper costume for a 30-ft. dragon (although the Dragonbane LARP, planned to take place in July 2006 in Sweden, is attempting to build a dragon using a robotic chassis build on top of a 10-ton forestry engine), however, it is relatively easier to create one for an orc. In general, there are two ways to handle this problem: (a) accept the limits of the physical world, or (b) simulate the fictional element. For example, although for technical and safety reasons, participants playing wizards cannot use fireball-spell simulations, a water balloon still requires throwing skill and furthermore generates a nice area effect splash. More rules-based and abstract resolution techniques can be applied. These considerations do not apply to MMORPGs where any conceivable world setting and effect can be conveyed, limited only by the abilities of the underlying game technology.

Recent innovations in game forms, such as augmented/mixed reality gaming, mobile gaming, transreality gaming, and full-immersion virtual reality (VR; e.g., in CAVE environments), appear to offer interesting new pathways to combining technologies with LARPs and combining the virtual and physical game spaces (e.g., Lindley, 2004; Magerkurth, Memisoglu, Engelke, \& Streitz, 2004).

\section{Storytelling in LARPs}

Storytelling in LARPs is constrained by the choice of approach to player objectives and story development, as discussed above, as well as the physical nature of the play. As in PnP RPGs, LARPS have three types of game space: (a) the shared play space, which encompasses the fictional world setting and the interactions occurring in-game; (b) the shared game space, which includes the actual environment of the players, any metagame layers, and the shared play space (i.e., both the in-game and off-game space; in LARPs, shared game space is used to differentiate between the game and the real world outside the physical parameters of the game); and (c) the subjective game space, as it is perceived by each participant.

The shared game space has somewhat blurred margins with the real world outside the game space; for example, players can break from the game and go get a pizza outside the designated gaming area, retaining their costumes and even acting in-character. This blurring of the edges of the shared game space leads to the possibility of real-life influence on the game: (a) A player could break a leg while eating on the way back to the gaming area, thus being removed from the game, possibly upsetting game flow. (b) Real-life issues such as relationships or enmities among participants can be brought 
into the shared game space, increasing the chance of conflicts arising both in-game and off-game. These types of personal problems are easier to control in PnP RPGs and will often lead to a game not being initiated at all. Because LARPs can have thousands of players who are unknown to the organizers/GMs, such problems can be imported. (c) Players can mistake the actions of other players to have an out-of-game significance (e.g., professions of love or similar). These problems emphasize the need for a solid fictional contract and premise for a LARP, especially one of large size.

Kim (2003) defined two general paradigms for storytelling in RPGs: (a) that the narrative is formed by the expressed communication of the players and GMs in the shared play space and (b) that the narrative is formed by the perceived story of each individual player. Both of these paradigms are applicable to LARPs, however, there appears to be some basis for defining the story of a LARP as a multilayered narrative: From the individual perspective, each player and GM perceives a given narrative based on what they have been involved with during the LARP. Similarly, a particular group of players who have spent most or all of the LARP together will have some shared elements unique to them. Finally, the entire LARP itself, if recorded, would form a multilinear narrative that would, however, be different from the perceived story of each individual player or group of players.

A LARP is, in general, the reverse of the RPG situation. Each player receives the same sensory input (although they can be in different physical locations), and the actions of the player have an autonomous legitimacy because they are (commonly) unguided by a GM. LARP players can therefore be seen as perceivers of individual story lines in a narrative matrix defined by the shared game space. Costikyan (1998) noted that "it would be . . . accurate to visualise a LARP as an ever-shifting hedgerow of converging and splintering plot threads, each one propelled by the engine of an autonomous player-character, but each one initially based on a perception of the physical game environment that is in total harmony with the perception of every other participant." In open-ended LARPs, where storytelling is decentralized, there is room for individual goals and initiative, and players can act independently of any other player. In this line of thinking, the sum of subjective game spaces gives rise to the narrative of a LARP.

\section{Information Technology in LARP}

Communication technologies can support the organizational processes of LARPs, ensuring information flow from GMs to players and facilitating the length and quality of actual in-game time. The usage of information technologies ranges from implementing radio communication and sensors in the games themselves to using chat boards and other online communication methods to handle organization, in-between session arrangements, and so forth. Similarly, online multimedia are used to keep players active between sessions and preserve their interest and the sense of community (Hartzell, 2002). 
LARPs form a testing ground for pervasive gaming technologies (e.g., embedded and ubiquitous computers) and technologies to aid social interaction. These properties of LARPs have resulted in a dawning research interest and exploration (e.g., Falk \& Davenport, 2004; Hartzell, 2002; Rój, 2004; Schneider \& Kortuem, 2001; Söderberg et al., 2003) of the activity. Schneider and Kortuem (2001) developed a LARP version of the board game Clue (2003 Hasbro Games), which used specially programmed PDAs to support the clue finding and exchange process of the players, in a research study of how to support player interactions in pervasive gaming environments such as LARPs. Söderberg et al. (2003) studied LARPs as an application domain for ubiquitous computing. LARPs provide tough testing conditions for ambient and embedded computer technologies, as well as demanding design requirements: A wide range of players must be able to understand and use the equipment with ease. The authors also address the use of ubiquitous computing in assisting development of player-generated, location-based content and implementations of the content without disturbing the game flow, the use of information technologies to aid the GMs in gathering and spreading information, and so on.

Falk and Davenport (2004) examined the potential that LARPs could have for pervasive gaming. They postulated that LARPs offer a fully immersive experience by providing a tangible and distributed interface to a gaming activity that is "emergent, improvised, collaboratively and socially created, and [has] the immediacy of personal experience" (p. 1), making LARPs appear the ideal of interactive entertainment. Falk and Davenport pointed to a trend in the development of electronic entertainment technologies with application for games and interactive narratives set in participation and interaction with the physical (real) world. This approach was used by Christensen, Jørgensen, and Jørgensen (2005), who developed a model for a hybrid MMORPG and LARP using usability methodology. Their case study emphasized the importance of embodiment, concurrency in actions, and rich communication as central factors for the successful transfer between the MMORPG and LARP components of the hybrid game.

\section{Discussion and Conclusion}

\section{Relating the Model to Forms and Cultures of LARP Play}

The model presented in this article outlines the various control functions that can exist in LARPs and provides a means of LARP classification via these functions. The model includes any form of LARP, and as a means for LARP classification, any contemporary LARP should be covered by the model.

It is important to realize that the general model does not detail the level to which the various control functions are used in a specific LARP. It lists the total range of functions that are available, not the level to which these are integrated. The level of control integration is discussed under the relevant sections and forms a basis for defining a LARP typology. 
For example, some LARPs use player characters that limit the range of possible behaviors/reactions in players, whereas others encourage characters that are more flexible. The character generation control function is still present in both cases and can be used to categorize the two types of LARPs.

In the LARP community worldwide, there is a general tendency to separate LARPs into authorial/GM-driven (scenario author and GMs have a substantial presence and temporal control) and distributed/player-driven (control is mostly distributed among the players; GMs may not be present) categories. Very broadly speaking, the former is very typical of LARPs in North America and Europe, whereas the latter is common in Scandinavia and Australia.

This article uses the concept of GMs to tie the control functions to the actual games, however, this does not mean that it is not applicable to player-driven LARPs. As discussed above, the actual presence and function of GMs in LARPs can vary substantially. This means that the level of control being exerted can rest with GMs or, conversely, be very distributed among the participants. For example, open LARPs generally distribute the in-game control to the players to a substantial degree. In player-driven LARP forms, the in-game control can be distributed completely among the participants; however, the different forms of control (see Figure 1) are still very much present, as discussed above. In LARPs without a specified GM capacity, in general, certain players will have specific responsibilities (e.g., managing a specific event). In these cases, the role of being GM could be said to be interchangeable, fluctuating between the participant players. It is here suggested that the term fluctuating GM is used to describe this form of live control in LARPs.

\section{Using LARP Experiences in MMORPG Design and Management}

LARPs are closely related to MMORPGs and provide both a source of ideas to be applied to MMORPGs and an arena to try ideas in large participant interactive narratives without the need for extensive software implementation. The LARP community has two decades of experience in delivering the same kind of interactive experiences the electronic games offer. At present, however, the knowledge base of LARPs is severely limited, and the vast majority of knowledge is assembled outside the academic framework. LARPs are also of interest as a venue for the application of mobile technology to interactive gaming.

In this article, a classification scheme for LARPs has been presented. This scheme demonstrates the range of possible game types that exist. This is not supposed to be the final word in the area; however, it should provide a starting point for further investigation and discussion. The total range of LARP types possible by combining the various features included in the classification presented here result in thousands of options. However, most of these will feature insignificant differences or rarely be used in practice. The next step in developing a classification model would therefore be to conduct more thorough surveys of LARPs and empirical experimentation. The 
surveys conducted in connection with this study suggest that further work in this regard will result in a limited number of overall types of LARP, with very similar features. Because of the similarities between large-scale LARPs and MMORPGs, and their relevance to interactive narrative in general, there is incentive to investigate these games in more detail. Such research would probably take the form of extensive observation of LARP play and interviews with the participants. Possible venues for further research include, but are not limited to, the following:

1. Narrative control: The amount of narrative control and how it is maintained varies substantially between LARP types, as described above. The implications of these variations in terms of authorial and GM effort, player appreciation, and character interaction require detailed investigation. The role-playing activity can be regarded as made up of several layers, including game layers, which include the actual rules (game mechanics) and are objective and visible; and the metagame layers, which cover subjective resolution mechanics, group contracts, and the act of role playing itself. These two can be further subdivided into, for example, near game, near metagame, and so on (Gleichman, 2002). This layered structure is important in a LARP in delineating the narrative control. The control exerted within each layer can vary between players and between players and the GMs. This means that control can vary not only among the players and GMs but also among the different layers. How a measure of narrative control or structure is maintained with limited GM resources and how the players perceive the narratives remain largely unexplored, as do questions such as how a LARP is perceived by a player and whether this is comparable with a MMORPG situation.

2. Differences between $\mathrm{PvP}$ and player versus environment $(\mathrm{PvE})$ types of play: LARPs can feature both PvP and PvE types of play. The effects of using either of these in relation to promoting certain types of behavior, style of play, and narrative development should be comparable with the MMORPG situation and possibly provide useful insights into these issues.

3. Trust issues: The fact that the player cannot hide behind a virtual account in a LARP, as in a MMORPG, possibly means that punishment for breaking rules and similar behavior is easier to mete out. This may be the primary reason that trust issues appear to play a significantly smaller role in LARPs than in MMORPGs. However, it is possible that other features factor in. The uncovering of these could be used to address trust problems in MMORPGs.

4. Promoting role play and good behavior: Lack of role playing (in general and in particular on those MMORPG servers dedicated to role playing, normally titled "RP servers," e.g., in World of Warcraft), grief behavior, and similar issues appear to be less of a problem in LARPs than in MMORPGs. This is probably partly due to the physical dimension, which prevents certain types of negative behavior. However, grief play, for example, remains very possible, and it would be of interest to investigate how LARPs handle these issues.

5. Communication of world setting and the fictional contract with the players: Largescale LARPs have for years been exposed to the problem of communicating a fictional world setting to players without imparting too high a workload on the players 
nor GMs/authors. LARPs use a combination of providing information pregame and integrating the player in the game world as the game progresses, and the way this is done provides a venue for exploration in relation to MMORPGs, where player introduction can be very limited (e.g., World of Warcraft).

6. GM in-game communication and rules regulation: LARP GMs have developed various ways of communicating in-game that might offer insights as to improving GM communication flow in MMORPGs.

Finally, although an apparently hitherto untried approach, LARP functionality could be used as the basis for presence experimentation in full VR, for example, using linked or multiuser CAVE systems in conjunction with motion capture tools or stereo projectors. There is some evidence to suggest that each person's sense of presence depends on their psychological make-up (Pimentel \& Teixeira, 1994). If this is so, there may not be a virtual reality experience convincing to all users. VR-based LARPs could form a testing ground for experiments into this subject. In relation to this subject, the development of combined full-VR LARP/MMORPG games forms an interesting research pathway, although such game forms are still only a theoretical possibility.

\section{References}

Bøckman, P., \& Hutchison, R. (2005). Dissecting LARP (Collected papers for Knutepunkt 2005). Norway: Grimshei trykkeri AS.

Christensen, L. J., Jørgensen, T.T.-Y., \& Jørgensen, A. H. (2005). Developing a hybrid of MMORPG and LARP using usability methods: The case of Takkar. In Proceedings of the 2005 DiGRA Conference. Retrieved September 26, 2005, from http://www.digra.org/dl/db/05150.45031

Costikyan, G. (1998). Don't be a vidiot-What computer game designers can learn from non-electronic games. Retrieved September 26, 2005, from http://www.costik.com/vidiot.html

Darlington, S. (1998-1999). A history of role-playing. In Places to go, people to be (Parts 1-9). Retrieved September 26, 2005, from http://ptgptb.org/0001/history1.html

Dollar, M. (2002). LRP in the U.K. The LARPer, 1(3). Retrieved September 26, 2005, from http://www .thelarper.org/UK_LRP.html

Edwards, R. (2001). GNS and other matters of role-playing theory. Retrieved September 26, 2005, from http://www.indie-rpgs.com/articles/1/

Emrick, T. E. (2001). A beginner's guide to LARP. Retrieved September 26, 2005, from www.geocities. com/timlarp/index.html

Falk, J., \& Davenport, G. (2004). Live role-playing games: Implications for pervasive gaming. In Proceedings of the ICEC 2004. Retrieved September 26, 2005, from http://springerlink.metapress.com

Fatland, E. (2005). Incentives as tools of LARP dramaturgy. In Dissecting LARP (Collected papers for Knutepunkt 2005, pp. 147-180). Norway: Grimshei trykkeri AS.

Fine, G. A. (2002). Shared fantasy: Role playing games as social worlds. Chicago: University of Chicago Press.

Gleichman, B. (2002). Layers of design. Retrieved September 26, 2005, from http://www.rpg.net/news+ reviews/columns/elements11dec02.html

Hartzell, E. (2002). Techno twinkies and you-LARPing in the information age. LARPer, 2(1). Retrieved September 26, 2005, from http://www.thelarper.org/techno_twinkies.html

Kim, J. H. (2003). Story and narrative paradigms in role-playing games. Retrieved September 26, 2005, from http://www.darkshire.net/ jhkim/rpg/theory/narrative/paradigms.html 
Lindley, C. A. (2004). Trans-reality gaming. In Proceedings of the 2 nd annual International Workshop in Computer Game Design and Technology. Liverpool, UK: Liverpool John Moores University.

Logas, H. (2004). The Taoist storyteller. Pleasures of the Flesh, 5. Retrieved September 26, 2005, from http://www.skotos.net/articles/pleasures5.phtml

Louchart, S., \& Aylett, R. (2003). Solving the narrative paradox in VEs-Lessons from RPGs. In Proceedings of the 2003 IVA. Retrieved September 26, 2005, from http://www.macs.hw.ac.uk/ ruth/ Papers/narrative/IVA03-Louchart-Aylett.pdf

Lynch, S. (2000). Border dispute: Drawing the line between LARP and tabletop gaming, or a tale of two role playing modes. Retrieved September 26, 2005, from http://www.rpg.net/news+reviews/columns/ Live_Wires_Scott_12_1.html

Mackay, D. (2001). The fantasy role-playing game: A new performing art. Jefferson, NC: McFarland and Company.

Magerkurth, C., Memisoglu, M., Engelke, T., \& Streitz, N. (2004). Towards the next generation of tabletop gaming experiences. ACM International Conference Proceeding Series, 62, 73-80.

Montola, M., \& Stenros, J. (Eds.). (2004). Beyond role and play-Tools, toys and theory for harnessing the imagination (for Solmukohta 2004). Helsinki, Finland: Ropecon Ry.

Murray, J. (1998). Hamlet on the holodeck. Cambridge, MA: MIT Press.

Peinado, F., \& Gervás, P. (2004). Transferring game mastering laws to interactive digital storytelling. In Proceedings of the 2nd International Conference on Technologies for Interactive Digital Storytelling and Entertainment. Retrieved September 26, 2005, from http://gaia.sip.ucm.es/grupo/publications/ 2004-peinado-transferring.pdf

Pimentel, K., \& Teixeira, K. (1994). Virtual reality: Through the new looking glass. New York: Windcrest.

Rój, M. (2004). Smart artifacts as a key component of pervasive games. In Proceedings of the Third International Conference on Pervasive Gaming: Pervasive 2004. Retrieved September 26, 2005, from http://www.ipsi.fraunhofer.de/ambiente/pervasivegaming/papers/manet-based-game.ps

Salen, K., \& Zimmerman, E. (2003). Rules of play: Game design fundamentals. Cambridge, MA: MIT Press.

Schneider, J., \& Kortuem, G. (2001). How to host a pervasive game: Supporting face-to-face interactions in live-action roleplaying. In Proceedings from the Workshop on Designing Ubiquitous Computing Games, Ubicomp. Retrieved September 26, 2005, from http://www.cs.uoregon.edu/research/wearables/ Papers/how2host.pdf

Söderberg, J., Waern, A., \& Åkesson, K.-P. (2004). Enhanced reality live role playing. In Proceedings of the Second International Conference on Pervasive Computing: Pervasive 2004. Retrieved September 26, 2005, from http://www.sics.se/ kalle/published/Pervasive2004/Enhanced\%20Reality\%20Live\%20 Role\%20 Playing.pdf

Tychsen, A., Hithchens, M., Brolund, T. and Kavakli, M. (2005). The game master. Proceedings of the 2005 Interactive Entertainment Conference, 123, 215-222.

Anders Tychsen is a games journalist and $\mathrm{PhD}$ candidate at the Department of Computing at Macquarie University in Sydney, where he operates from within the Interactive Systems and Virtual Reality Research Group. His work centers on communication, storytelling in and design of multi- and massively multiplayer games, especially of the role-playing variant. His research reaches however broadly to encompass most aspects of communication, immersion, narratives, and interactive entertainment, within the overall framework of computer- and role-playing games. He also works with games design, focusing on the process of world generation and development (e.g., ecological systems) in MMORPGs.

Michael Hitchens (PhD, Newcastle, 1991) is a senior lecturer and the director of teaching and learning in the Division of Information and Communication Sciences at Macquarie University in Sydney. His research interests in the area of games include storytelling and player experience. 
Thea Brolund is a multiple-award winning PhD Candidate at the Department of Environmental Sciences at the University of Technology in Sydney. Her research is focused on large-scale variations in the distribution of coral reef faunas. She is also actively engaged in games research, notably massively multiplayer games. Her focus is on the development of methods and models for the development of believable natural ecologies encouraging emergent behavior in Massively Multiplayer Online Games, as well as methods for controlling player interaction with the these systems.

Manolya Kavakli is a senior lecturer at the Department of Computing at Macquarie University in Sydney. Her main areas of research are interactive systems development and methodologies for design computing, virtual reality, and games. She is the recipient of a number of international awards and fellowships. She worked as the course coordinator of the first Computer Science (Games Technology) degree in Australia in 2002. In 2003, she established a Virtual Reality Lab for fostering graphics research at Macquarie University, which led to the creation of a research team, the Interactive Systems and Virtual Reality (ISVR) Research Group with 30 interdisciplinary members receiving a large number of grants. She has recently built a CAVE (immersive projection system) at the ISVR Lab, in a strategic partnership with VR Solutions. 